\title{
Effect of Magnetic Field on Peristaltic Flow of Williamson Fluid in a Symmetric Channel
}

\author{
Khalid AL-Qaissy* Ahmed M. Abdulhadi, \\ Department of Mathematics, College of Science, University of Baghdad, Baghdad, Iraq
}

\begin{abstract}
This paper deals with the influence of magnetic field on peristaltic flow of an incompressible Williamson fluid in a symmetric channel with heat and mass transfer. Convective conditions of heat and mass transfer are employed. Viscous dissipation and Joule heating are taken into consideration.Channel walls have compliant properties. Analysis has been carried out through long wavelength and low Reynolds number approach. Resulting problems are solved for small Weissenberg number. Impacts of variables reflecting the salient features of wall properties, concentration and heat transfer coefficient are pointed out. Trapping phenomenon is also analyzed.
\end{abstract}

Keywords: Williamson fluid, compliant walls.

\section{Introduction}

Peristalsis is the form of fluid transport due to wave travelling along the walls of an inextensible tube/channel. This type of rhythmic contraction provides the basis of peristaltic pumps to transport fluid through different tubular parts without any direct contact (see studies [1]). [2] also discussed the influence of rotationand initial stresses on peristaltic flow of fourth grade fluid in an asymmetric channel. The peristaltic flow of nano fluid through a porous medium with mixed convection is analyzed by Nowar [3]. Kothandapani and Prakash [4] studied the combined effects of radiation and magnetic field on peristaltic transport of nanofluid. The studies related to peristalsis with heat and mass transfer for Newtonian fluid Have been structured in refs. [5, 6] . [7] discussed the peristaltic flow in a vertical channel filled with nanofluid. Peristaltic transport of fourth grade fluid is numerically discussed by Mustafa et. al.[8] . Kothand apani and Prakash [9] analyzed the peristaltic transport of nanofluid with thermal radiation and magnetic field in a tapered channel. Mehmoodetal. [10] considered the partial slip effects on peristaltic transport in a channel with heat and mass transfer effects. Mathematical analysis has been carried out in the presence of magnetic field. In all of the above mentioned studies lubrication approachhas been utilized to simplify the problems. It is seen that heat transfer in peristalsis has pivoted role.

\section{Basic Equations}

The continuity, momentum, energy and concentration equation in the presence of viscous dissipation and joule heating are given by

$$
\operatorname{div} V=0
$$

$$
\begin{array}{r}
\rho \frac{d V}{d t}=\operatorname{div} \tau^{\prime}+\rho f(\mathbf{2}) \\
\rho C_{p} \frac{d T}{d t}=k \nabla^{2} T+\frac{D K_{T}}{C_{s}}+\frac{1}{\sigma}(J \cdot J)(\mathbf{3})
\end{array}
$$

Where $V$ is velocity vector, $\tau^{\prime}$ the Cauchy stress tenser, $\rho$ the body force, $\mathrm{d} / \mathrm{dt}$ the material time derivative , $C_{p}$ the special heat at constant volume,$J$ the current density , $T$ the temperature of fluid $D$ thecoefficient of mass diffusivity, $k$ the thermal diffusion ratio,$C_{S}$ the concentration susceptibility. The constitutive equations fluid is $\tau^{\prime}=-p I+s(\mathbf{4})$

$s=\mu_{0}[(1+\Gamma \dot{\gamma})] A_{1}(\mathbf{5})$

Where $p$ is the pressure, $s$ the extra stress tensor, $\mu_{0}$ the zero shear rate viscosity, $\Gamma$ the time constant and $\dot{\gamma}$ is

$$
\dot{\gamma}=\sqrt{\frac{1}{2} \pi},(\mathbf{6})
$$

In which $\pi=\operatorname{tr}\left(A_{1}{ }^{2}\right)$ where $A_{1}=\nabla \vec{V}+(\nabla \vec{V})^{T}$.

Here Eq.(5) corresponds to the viscous fluid when $\Gamma=0$.

\section{Mathematical Formulation}

Conseder an incompressible magnetohydrodynamic (MHD) flow of Williamson fluid in a symmetric channel of width $2 d_{1}$. Here $x$-axis is taken along the length of channel and y-axis transvers to it (see Fig 1). The induced magnetic field is neglected by assuming a very small magnetic Reynolds number .Also the electric field is taken absent.the flow is generate by sinusoidal waves propagating along the compliant walls of channel: 
$\bar{y}= \pm \bar{\eta}(\bar{x}, \bar{t})= \pm\left[d+m \bar{x}+a \sin \frac{2 \pi}{\lambda}(\bar{x}-c \bar{t})\right](7)$

Where $a$ is the wave amplitude,$\lambda$ the wavelength , c the wave

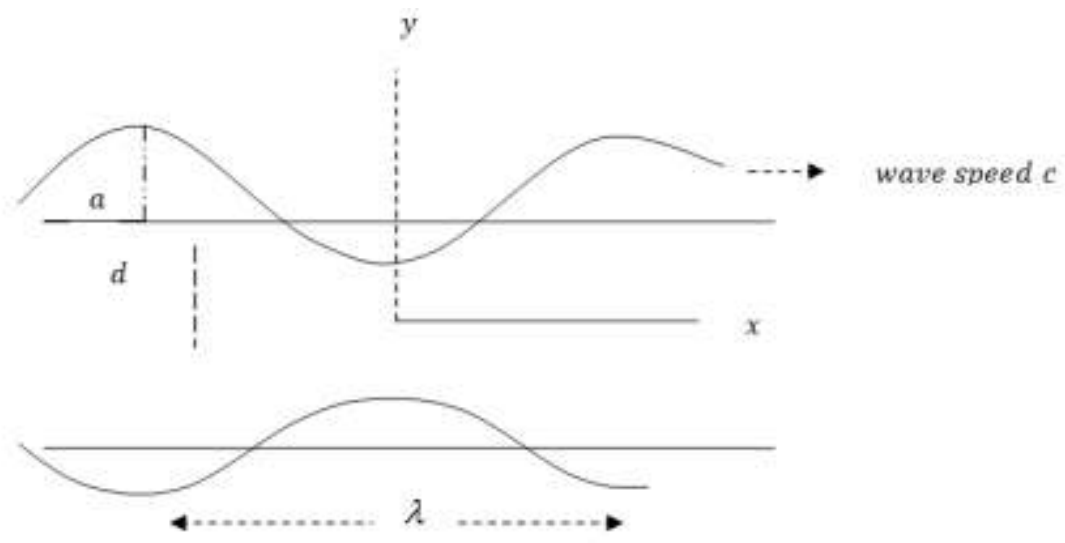

Figure 1 Diagrammatic of the problem

In wave frame, the equations which govern the flow are given by:

$\frac{\partial \bar{u}}{\partial \bar{x}}+\frac{\partial \bar{v}}{\partial \bar{y}}=0(\mathbf{8})$

$\rho\left(\bar{u} \frac{\partial \bar{u}}{\partial \bar{x}}+\bar{u} \frac{\partial \bar{v}}{\partial \bar{y}}\right)=-\frac{\partial \bar{p}}{\partial \bar{x}}+\frac{\partial}{\partial \bar{x}}\left[2 \mu_{0}(1+\Gamma \overline{\dot{\gamma}}) \frac{\partial \bar{u}}{\partial \bar{x}}\right]$

$+\frac{\partial}{\partial \bar{y}}\left[\mu_{0}(1+\Gamma \overline{\dot{\gamma}})\left(\frac{\partial \bar{u}}{\partial \bar{y}}+\frac{\partial \bar{v}}{\partial \bar{x}}\right)\right]-\sigma \beta_{0}{ }^{2} \bar{u}-\frac{\mu}{k} \bar{u}(\mathbf{9})$

$\rho\left(\bar{v} \frac{\partial \bar{u}}{\partial \bar{x}}+\bar{v} \frac{\partial \bar{v}}{\partial \bar{y}}\right)=-\frac{\partial \bar{p}}{\partial \bar{y}}+\frac{\partial}{\partial \bar{x}}\left[\mu_{0}(1+\Gamma \bar{\gamma})\left(\frac{\partial \bar{v}}{\partial \bar{x}}+\frac{\partial \bar{u}}{\partial \bar{y}}\right)\right]$

$+\frac{\partial}{\partial \bar{y}}\left[2 \mu_{0}(1+\Gamma \bar{\gamma}) \frac{\partial \bar{v}}{\partial \bar{y}}\right]+\frac{\mu}{k} \bar{v}(\mathbf{1 0})$

$\rho c_{p} \frac{d T}{d \bar{t}}=k\left[\frac{\partial^{2} T}{\partial \bar{x}^{2}}+\frac{\partial^{2} T}{\partial \bar{y}^{2}}\right]+$

$\mu_{0}(1+\Gamma \bar{\gamma})\left[\left(\frac{\partial \bar{v}}{\partial \bar{x}}+\frac{\partial \bar{u}}{\partial \bar{y}}\right)^{2}+2\left(\frac{\partial \bar{u}}{\partial \bar{x}}\right)^{2}+2\left(\frac{\partial \bar{v}}{\partial \bar{y}}\right)^{2}\right](\mathbf{1 1})$

where $\rho$ is the density, $B_{0}$ is the strength of the magnetic field, $\bar{p}$ is the pressure, $(\bar{u}, \bar{v})$ are the components of the velocity vector $\vec{V}$ in the $\bar{x}$ and $\bar{y}$ directions respectively and $\sigma$ the electrical conductivity. The corresponding boundary condition are given by

$\bar{u}= \pm \bar{\beta} \frac{\partial \bar{u}}{\partial \bar{y}} a t \bar{y}= \pm \bar{\eta}(\mathbf{1 2})$

$\left[-\tau \frac{\partial^{3}}{\partial \bar{x}^{3}}+m \frac{\partial^{3}}{\partial \bar{x} \partial \bar{t}^{2}}+\tilde{d} \frac{\partial^{2}}{\partial \bar{x} \partial \bar{t}} B \frac{\partial^{5}}{\partial \bar{x}^{5}}+H \frac{\partial}{\partial \bar{x}}\right](\bar{\eta})=\frac{\partial}{\partial \bar{x}} 2 \mu_{0}(1+\Gamma \overline{\dot{\gamma}}) \frac{\partial \bar{u}}{\partial \bar{x}}+\frac{\partial}{\partial \bar{y}}\left[\mu_{0}(1+\Gamma \overline{\dot{\gamma}})\left(\frac{\partial \bar{v}}{\partial \bar{x}}+\frac{\partial \bar{u}}{\partial \bar{y}}\right)\right] a t \bar{y}= \pm \bar{\eta}(\mathbf{1 3})$

$T=T_{0} a t \bar{y}= \pm \bar{\eta}(\mathbf{1 4})$

In above equations $\tau$ is elastic tension in the membrane,$m$ the mass per unit area, $\tilde{d}$ the coefficient of viscous damping and $H$ the spring stiffness.

\section{Dimensionless Analysis}

Defining velocity component $u$ and $v$ in terms of stream function, introduce the dimensionless variables $x=\frac{\bar{x}}{\lambda}, y=\frac{\bar{y}}{d}, \epsilon=\frac{a}{d}, u=\frac{\bar{u}}{c}, v=\frac{\bar{v}}{c \delta}, p=\frac{d^{2} \bar{p}}{c \lambda \mu_{0}}, t=\frac{c \bar{t}}{\lambda}, \quad W e=\Gamma \frac{c}{a}, B r=\operatorname{Pr} E c, \operatorname{Pr}=$ $\mu O C P K, E C=c 2 T O C P, \quad E_{1}=-\tau d 3 \lambda 3 \mu O C, E 2=m c d 3 \lambda 3 \mu 0 \quad, \quad E 3=d 3 d \lambda 3 \mu 0$, $E_{4}=\frac{B d^{3}}{c \lambda^{3} \mu_{0}}, E_{5}=\frac{H d^{3}}{c \lambda \mu_{0}}, \operatorname{Re}=\frac{c d \rho}{\mu_{0}}, \overline{\dot{\gamma}}=\dot{\gamma} \frac{d}{c}, M=\sqrt{\frac{\sigma}{\mu_{0}}} \beta_{0} d, \dot{\dot{\gamma}}=\dot{\gamma} \frac{d}{c}, \theta=\frac{T-T_{0}}{T_{0}}(\mathbf{1 5})$

And use long wave length assumption $(\delta \ll 1)$ the notion equation and temperature equation can be written as $\frac{\partial^{2}}{\partial y^{2}}\left[(1+W e \dot{\gamma})\left(\frac{\partial^{2} \psi}{\partial y^{2}}\right)\right]-M^{2} \frac{\partial \psi}{\partial y}-\frac{1}{k} \frac{\partial \psi}{\partial y}(\mathbf{1 6})$

$\frac{\partial^{2} \theta}{\partial y^{2}}+B r(1+W e \dot{\gamma})\left(\frac{\partial^{2} \psi}{\partial y^{2}}\right)^{2}=0(\mathbf{1 7})$ 


$$
\begin{aligned}
& \frac{\partial \psi}{\partial y}= \pm \beta \frac{\partial^{2} \psi}{\partial y^{2}} \text { aty }= \pm \eta(\mathbf{1 8}) \\
& {\left[E_{1} \frac{\partial^{3}}{\partial x^{3}}+E_{2} \frac{\partial^{3}}{\partial x \partial t^{2}}+E_{3} \frac{\partial^{2}}{\partial x \partial t}+E_{4} \frac{\partial^{5}}{\partial x^{5}}+E_{5} \frac{\partial}{\partial x}+\right](\eta)=} \\
& \frac{\partial}{\partial y}\left[(1+W e \dot{\gamma})\left(\frac{\partial^{2} \psi}{\partial y^{2}}\right)\right] \text { aty }= \pm \eta(\mathbf{1 9}) \\
& \theta=0 \text { aty }= \pm \eta(\mathbf{2 0}) \\
& \dot{\gamma}=\frac{\partial^{2} \psi}{\partial y^{2}}(\mathbf{2 1}) \\
& \text { Here } \eta=1+\varepsilon \sin [2 \pi(x-t)]
\end{aligned}
$$

\section{Solution Procedure}

It seems difficult to solve Eqs.(16) to (21) in closed form . Thus we aim to find series solutions for small Weissenberg number and write

$\psi=\psi_{0}+W e \psi_{1}(\mathbf{2 2})$

$\theta=\theta_{0}+W e \theta_{1}(\mathbf{2 3})$

Here we have considered the zeroth and first order systems only due to the fact that the perturbation is taken small $(W e \ll 1)$ so that contrubation of higher order terms are negligible due to order analysis.

Zeroth-order system with its boundary conditions

Substitute (22) and (23) in to equations (16) to (21) and equating the coefficients of equal power of Weissenberg number we get zero and first order system and they are as :

$\frac{\partial^{4} \psi_{0}}{\partial y^{4}}-A^{2} \frac{\partial^{2} \psi_{0}}{\partial y^{2}}=0(\mathbf{2 4})$

$\left[E_{1} \frac{\partial^{3}}{\partial x^{3}}+E_{2} \frac{\partial^{3}}{\partial x \partial t^{2}}+E_{3} \frac{\partial^{2}}{\partial x \partial t}+E_{4} \frac{\partial^{5}}{\partial x^{5}}+E_{5} \frac{\partial}{\partial x}+\right](\eta)=$

$\frac{\partial}{\partial y}\left[\left(\frac{\partial^{2} \psi_{0}}{\partial y^{2}}\right)\right]$ aty $= \pm \eta(\mathbf{2 5})$

$\frac{\partial \psi_{0}}{\partial y}= \pm \beta \frac{\partial^{2} \psi_{0}}{\partial y^{2}}$ aty $= \pm \eta(\mathbf{2 6})$

$\frac{\partial^{2} \theta_{0}}{\partial y^{2}}+B r\left(\frac{\partial^{2} \psi_{0}}{\partial y^{2}}\right)^{2}=0(27)$

$\theta_{0}=0$ aty $= \pm \eta(\mathbf{2 8})$

Here $A^{2}=M^{2}+\frac{1}{k}$ and $\eta=1+\varepsilon \sin [2 \pi(x-t)]$.

\section{And the first-order system with its boundary conditions}

$$
\begin{aligned}
& \frac{\partial^{4} \psi_{1}}{\partial y^{4}}-A^{2} \frac{\partial^{2} \psi_{1}}{\partial y^{2}}+2\left(\frac{\partial^{3} \psi_{0}}{\partial y^{3}}\right)^{2}+2 \frac{\partial^{2} \psi_{0}}{\partial y^{2}} \frac{\partial^{4} \psi_{0}}{\partial y^{4}}=0 \text { (29) } \\
& \frac{\partial \psi_{1}}{\partial y}= \pm \beta \frac{\partial^{2} \psi_{1}}{\partial y^{2}} \text { aty }= \pm \eta(\mathbf{3 0}) \\
& 0=\left[\frac{\partial^{3} \psi_{1}}{\partial y^{3}}+2\left(\frac{\partial^{2} \psi_{0}}{\partial y^{2}}\right)\left(\frac{\partial^{3} \psi_{0}}{\partial y^{3}}\right)\right] \text { aty }= \pm \eta \mathbf{( 3 1 )} \\
& \frac{\partial^{2} \theta_{1}}{\partial y^{2}}+B r \frac{\partial^{2} \psi_{0}}{\partial y^{2}}\left(2 \frac{\partial^{2} \psi_{1}}{\partial y^{2}}+\left(\frac{\partial^{2} \psi_{0}}{\partial y^{2}}\right)^{2}\right)=0(\mathbf{3 2}) \\
& \theta_{1}=0 \text { aty }= \pm \eta(\mathbf{3 3}) \\
& \text { Here } A^{2}=M^{2}+\frac{1}{k} \text { and } \eta=1+\varepsilon \sin [2 \pi(x-t)] .
\end{aligned}
$$

Solve the zero and first order systems it is found them the stream function and temperature are given by

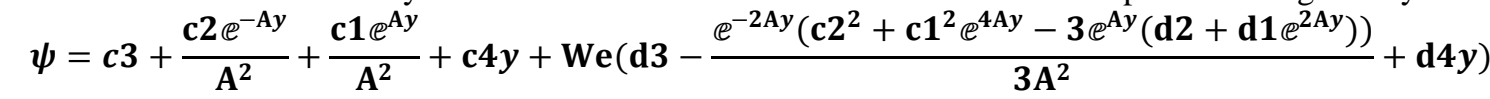

(34)

$\boldsymbol{\theta}=$

$$
\begin{aligned}
& \mathbf{b} 1+\mathbf{b} 2 y-\frac{\mathrm{Br}\left(\mathbf{c} 2^{2} e^{-2 \mathrm{~A} y}+\mathbf{c 1} \mathbf{1}^{2} e^{2 \mathrm{~A} y}+4 \mathbf{c 1} 1 \mathbf{c} 2 \mathrm{~A}^{2} y^{2}\right)}{4 \mathrm{~A}^{2}}+
\end{aligned}
$$

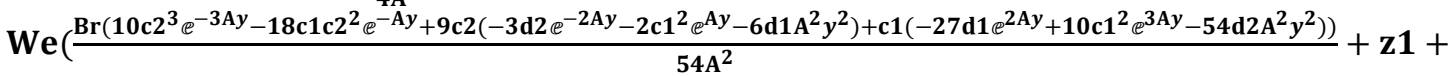

$$
\begin{aligned}
& \text { yz2 (35) }
\end{aligned}
$$


Here the constants $\boldsymbol{c} \mathbf{1}, \boldsymbol{c} \mathbf{2}, \boldsymbol{c} \mathbf{3}, \boldsymbol{c 4}, \boldsymbol{d} \mathbf{1}, \boldsymbol{d} \mathbf{2}, \boldsymbol{d} \mathbf{3}, \boldsymbol{d 4}, \boldsymbol{b} \mathbf{1}, \boldsymbol{b} \mathbf{2}, \boldsymbol{z} \mathbf{1}$ and $\mathbf{z} \mathbf{2}$ can be evaluated using MATHEMATICA

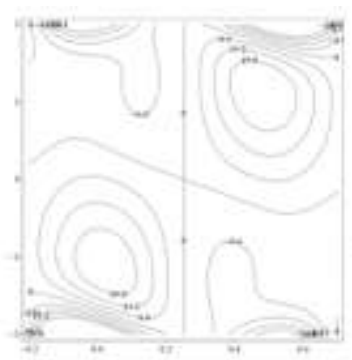

(a)

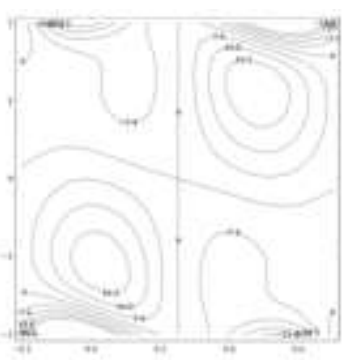

(b)

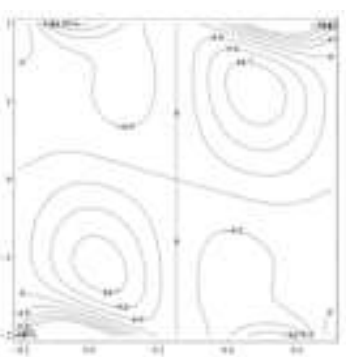

(c)

Figure 2. Stream line for different values of $E_{1}(a) E_{1}=0.5,(b) E_{1}=0.7,(c) E_{1}=0.8$ and the other parameters $E_{2}=0.2, E_{3}=0.1, E_{4}=0.05, E_{5}=0.3, W e=0.03, \varepsilon=0.2, t=0.02, M=1, m=0.01$, $\beta=0.01$.

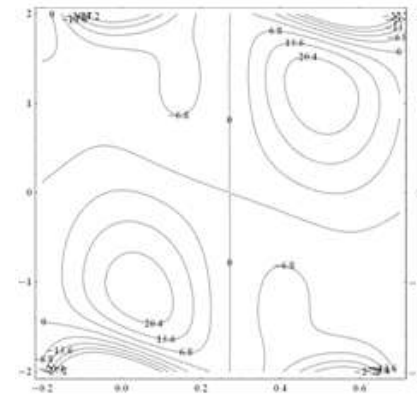

(a)

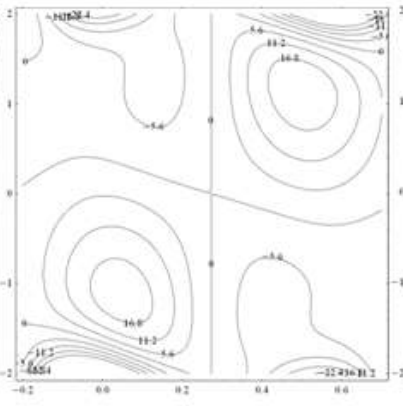

(b)

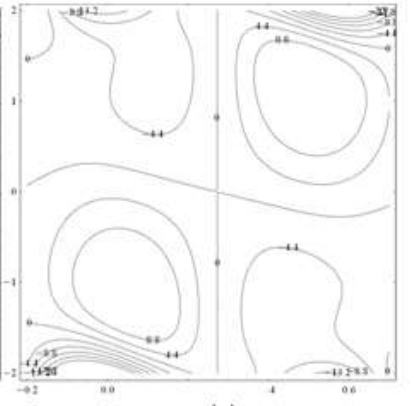

(c)

Figure 3. Stream line for different values of $E_{2}(a) E_{2}=0.2,(b) E_{2}=0.4,(c) E_{2}=0.6$ and the other parameters $E_{1}=0.5, E_{3}=0.1, E_{4}=0.05, E_{5}=0.3, W e=0.03, \varepsilon=0.2, t=0.02, M=1, m=$ $0.01, \beta=0.01$.

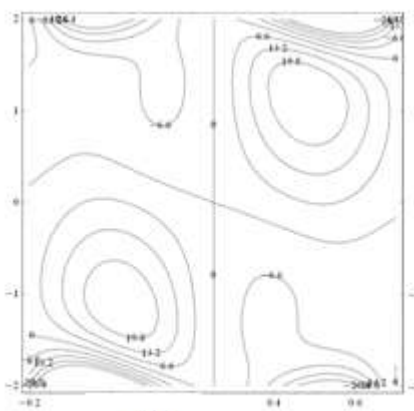

(a)

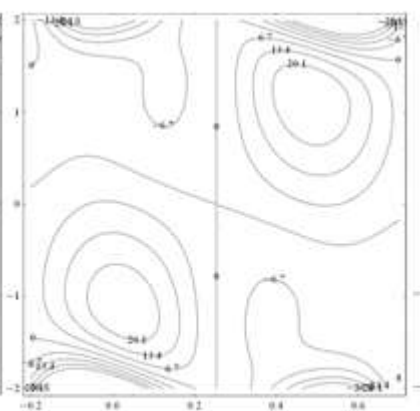

(b)

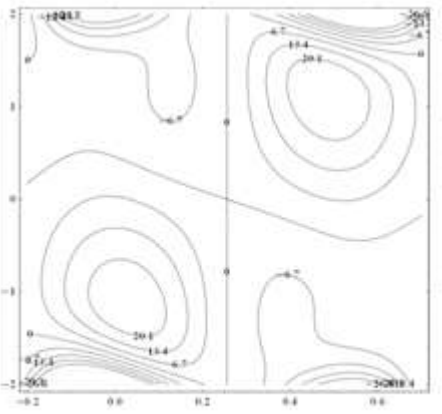

(c)

Figure 4. Stream line for different values of $E_{3}(a) E_{3}=0.1,(b) E_{3}=0.2,(c) E_{3}=0.3$ and the other parameters $E_{1}=0.5, E_{2}=0.2, E_{4}=0.05, E_{5}=0.3, W e=0.03, \varepsilon=0.2, t=0.02, M=1, m=$ $0.01, \beta=0.01$.

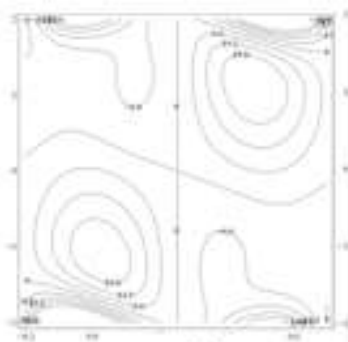

(a)

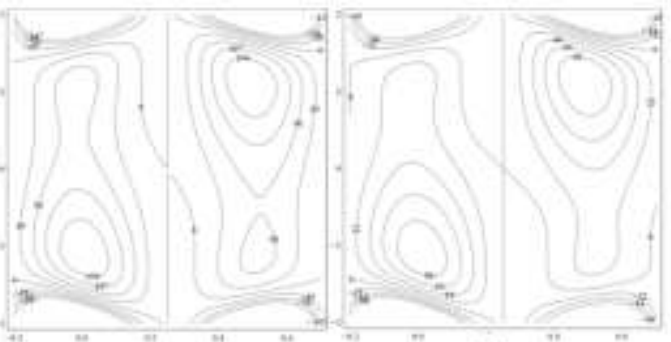

(b) (c) 
Figure 5. Stream line for different values of $E_{4}(a) E_{4}=0.05,(b) E_{4}=0.10,(c) E_{4}=0.15$ and the other parameters $E_{1}=0.5, E_{2}=0.2, E_{3}=0.1, E_{5}=0.3, W e=0.03, \varepsilon=0.2, t=0.02, M=1, m=0.01$, $\beta=0.01$.

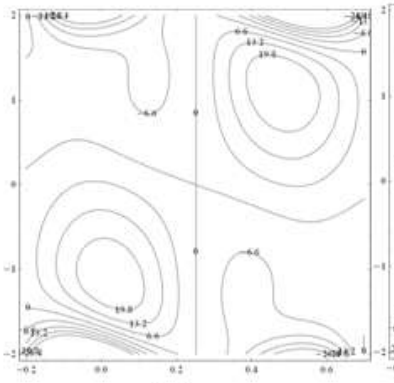

(a)

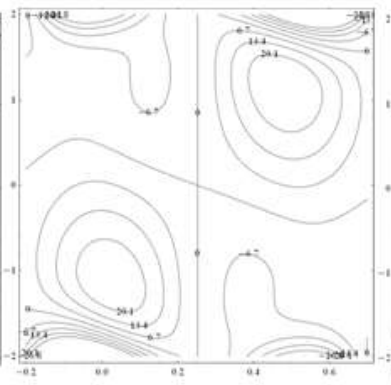

(b)

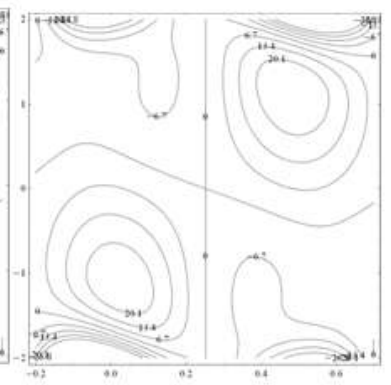

(c)

Figure 6.Stream line for different values of $E_{5}(a) E_{5}=0.3,(b) E_{5}=0.6,(c) E_{5}=0.9$ and the other parameters $E_{1}=0.5, E_{2}=0.2, E_{3}=0.1, E_{4}=0.05, W e=0.03, \varepsilon=0.2, t=0.02, M=1, m=$ $0.01, \beta=0.01$.

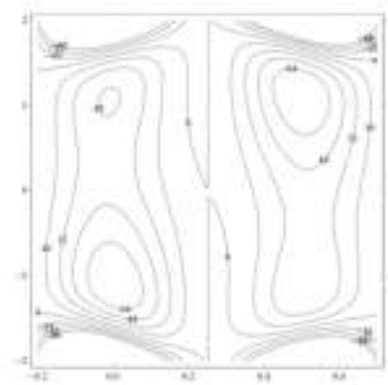

(a)

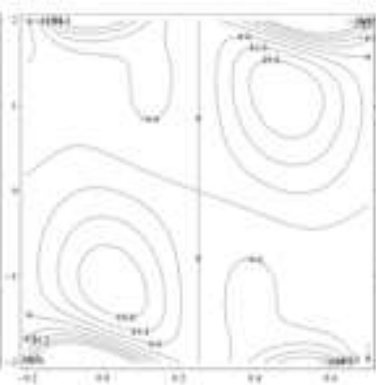

(b)

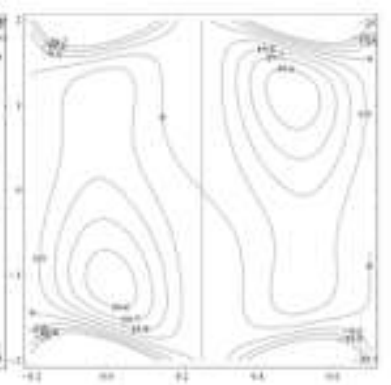

(c)

Figure 7.Stream line for different values of $W e(a) W e=0.03,(b) W e=0.09,(c) W e=0.18$ and the other parameters $E_{1}=0.5, E_{2}=0.2, E_{3}=0.1, E_{4}=0.05, E_{5}=0.3, \varepsilon=0.2, t=0.02, M=1, m=0.01$, $\beta=0.01$.

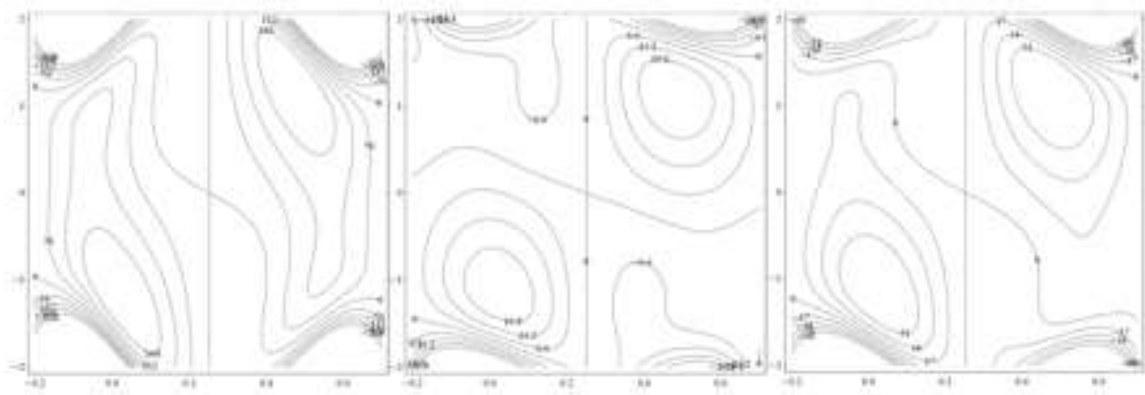

(a)

(b)

(c)

Figure 8. Stream line for different values of $\varepsilon(a) \varepsilon=0.2,(b) \varepsilon=0.4,(c) \varepsilon=0.8$ and the other parameters $E_{1}=0.5, E_{2}=0.2, E_{3}=0.1, E_{4}=0.05, E_{5}=0.3, W e=0.03, t=0.02, M=1, m=0.01, \beta=$ 0.01 .

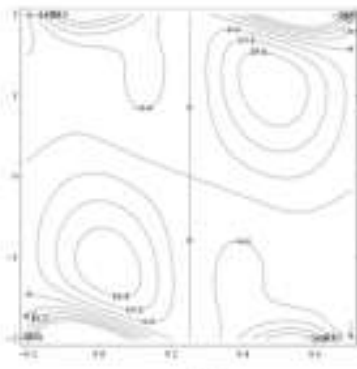

(a)

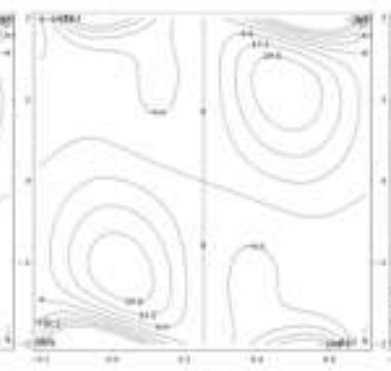

(b)

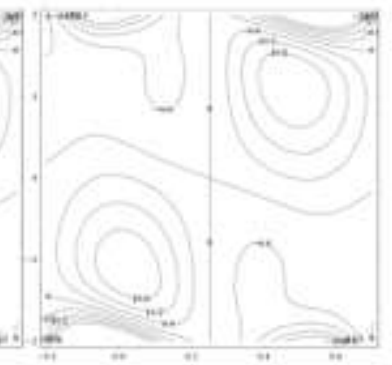

(c) 
Figure 9. Stream line for different values of $t(a) t=0.02,(b) t=0.04,(c) t=0.06$ and the other parameters $E_{1}=0.5, E_{2}=0.2, E_{3}=0.1, E_{4}=0.05, E_{5}=0.3, W e=0.03, \varepsilon=0.02, M=1, m=$ $0.01, \beta=0.01$.

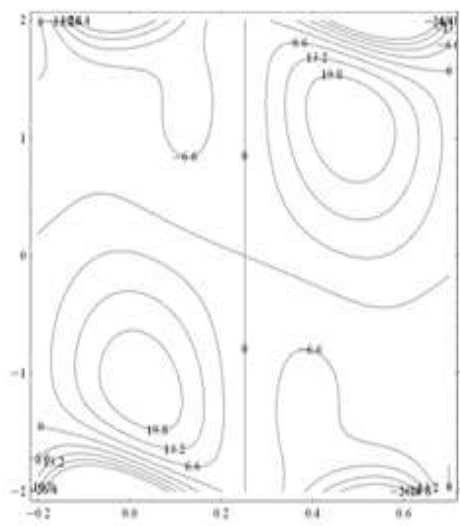

(a)

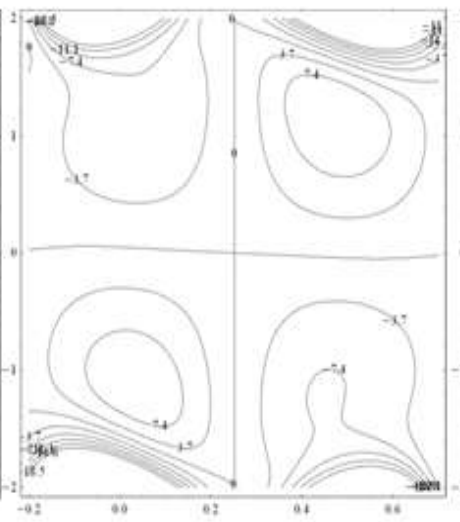

(b)

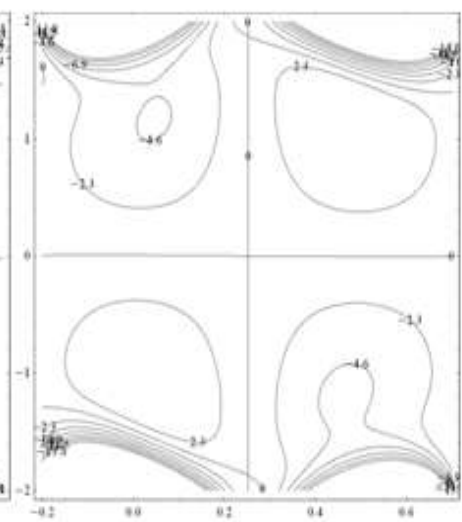

(c)

Figure 10. Stream line for different values of $M(a) M=1,(b) M=2,(c) M=3$ and the other parameters $E_{1}=0.5, E_{2}=0.2, E_{3}=0.1, E_{4}=0.05, E_{5}=0.3, W e=0.03, \varepsilon=0.2, t=0.02, m=0.01, \beta=0.01$

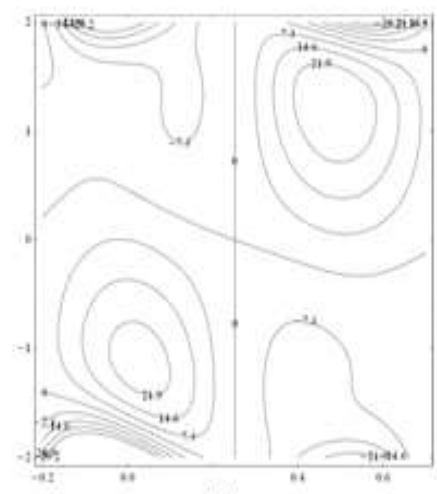

(a)

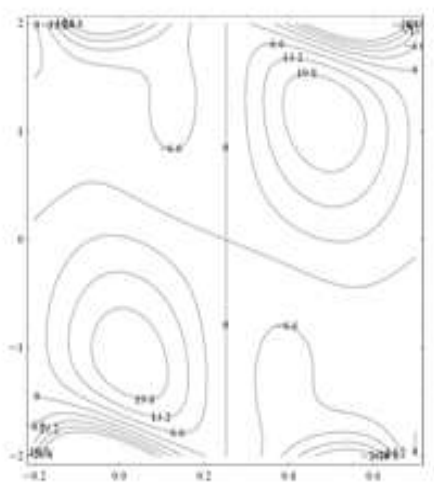

(b)

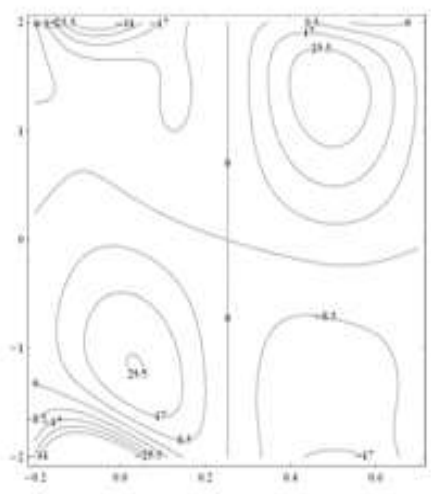

(c)

Figure 11.Stream line for different values of $m(a) m=0.01,(b) m=002,(c) m=0.03$ and the other parameters $E_{1}=0.5, E_{2}=0.2, E_{3}=0.1, E_{4}=0.05, E_{5}=0.3, W e=0.03, \varepsilon=0.2, t=0.02, M=$ $1, \beta=0.01$.

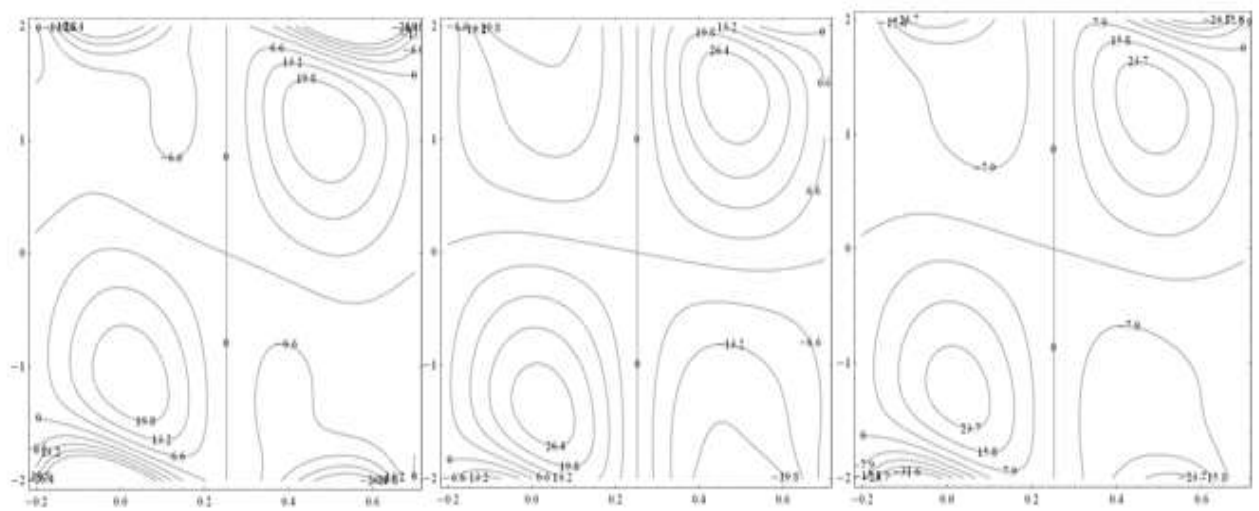

(a)

(b)

(c)

Figure 12. Stream line for different values of $\beta(a) \beta=0.01,(b) \beta=002,(c) \beta=0.03$ and the other parameters $E_{1}=0.5, E_{2}=0.2, E_{3}=0.1, E_{4}=0.05, E_{5}=0.3, W e=0.03, \varepsilon=0.2, t=0.02, M=1$, $m=0.01$. 

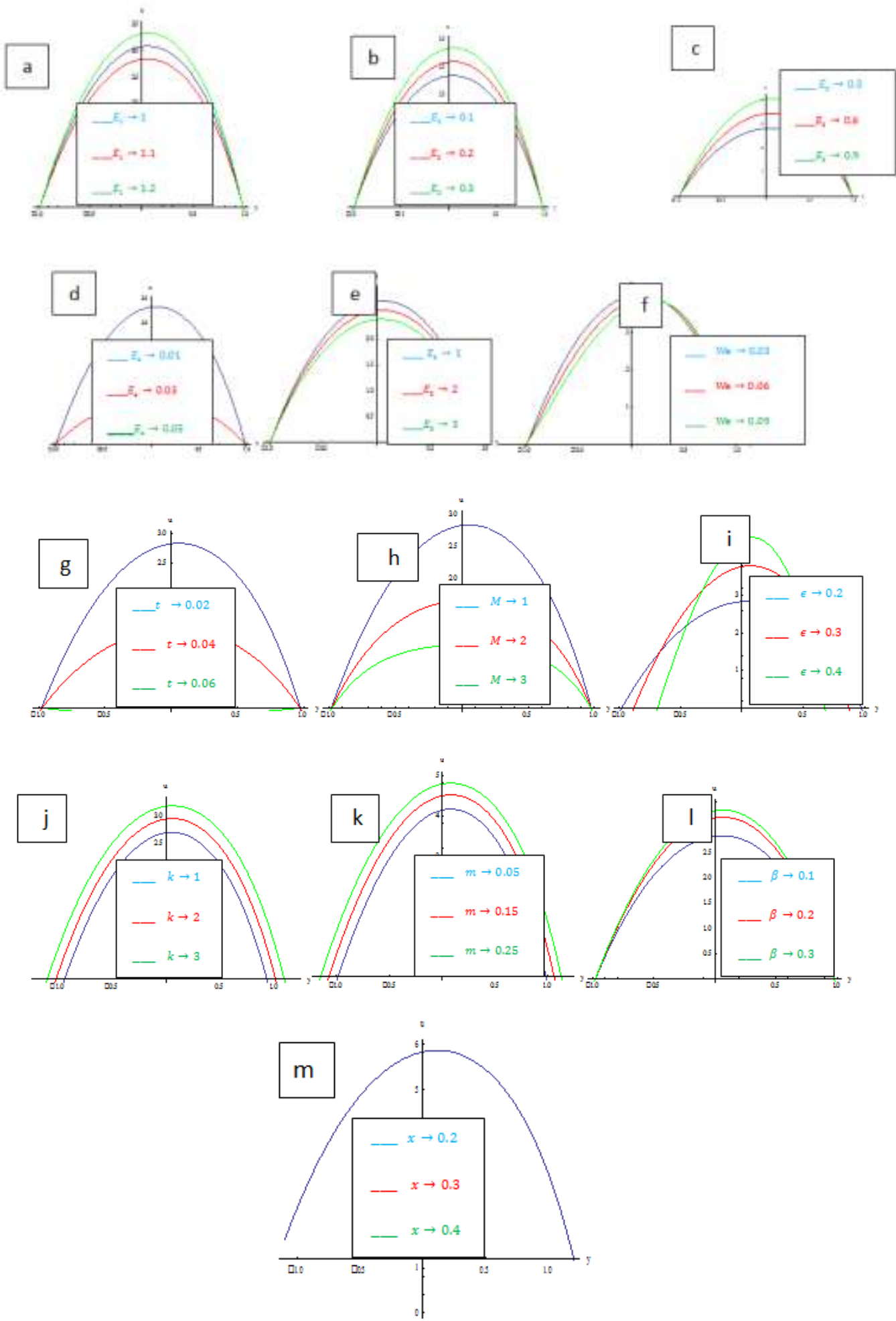

Figure 13.(a) Variation of $u$ with y for different values of $E_{1}$ at $E_{2}=0.3, E_{3}=0.3, E_{4}=0.01, E_{5}=0.01$, We $=0.03, \epsilon=0.2, t=0.02, M=1, k=1, m=0.1, \beta=0.1, x=0.8$. (b)- Variation of $u$ with y for different values of $E_{2}$ at $E_{1}=1, E_{3}=0.3, E_{4}=0.01, E_{5}=0.01$, We $=0.03, \epsilon=0.2, t=0.02, M=1$, $k=1, m=0.1, \beta=0.1, x=0.8$. (c)- Variation of $u$ with y for different values of $E_{2}$ at $E_{1}=1, E_{3}=$ $0.3, E_{4}=0.01, E_{5}=0.01, \mathrm{We}=0.03, \epsilon=0.2, t=0.02, M=1, k=1, m=0.1, \beta=0.1, x=0.8$. (d)- Variation of $u$ with y for different values of $E_{4}$ at $E_{1}=1, E_{2}=0.1, E_{3}=0.3, E_{5}=0.01$, We $=0.03$, 
$\epsilon=0.2, t=0.02, M=1, k=1, m=0.1, \beta=0.1, x=0.8$. (e)- Variation of uwith $\mathrm{y}$ for different values of $E_{5}$ at $E_{1}=1, E_{2}=0.1, E_{3}=0.3, E_{4}=0.01, \mathrm{We}=0.03, \epsilon=0.2, t=0.02, M=1, k=1$, $m=0.1, \beta=0.1, x=0.8$. (f)- Variation of $u$ with $\mathrm{y}$ for different values of Weat $E_{1}=1, E_{2}=0.1, E_{3}=$ $0.3, E_{4}=0.01 E_{5}=0.01, \epsilon=0.2, t=0.02, M=1, k=1, m=0.1, \beta=0.1, x=0.8$. (g)- Variation of $u$ with y for different values of $t$ at $E_{1}=1, E_{2}=0.1, E_{3}=0.3, E_{4}=0.01, E_{5}=0.01$, We $=0.03, \epsilon=$ $0.2, M=1, k=1, m=0.1, \beta=0.1, x=0.8$. (h)- Variation of $u$ with $\mathrm{y}$ for different values of $M$ at $E_{1}=1, E_{2}=0.1, E_{3}=0.3, E_{4}=0.01, E_{5}=0.01$, We $=0.03, \epsilon=0.2, t=0.02, k=1, m=0.1$,

$\beta=0.1, x=0.8$. (i)- Variation of $u$ with $\mathrm{y}$ for different values of $\epsilon$ at $E_{1}=1, E_{2}=0.1, E_{3}=0.3, E_{4}=$ $0.01, E_{5}=0.01$, We $=0.03, M=1, t=0.02, k=1, m=0.1, \beta=0.1, x=0.8$. (j)- Variation of $u$ with $\mathrm{y}$ for different values of $k$ at $E_{1}=1, E_{2}=0.1, E_{3}=0.3, E_{4}=0.01, E_{5}=0.01$, We $=0.03, \epsilon=$ $0.2, t=0.02, M=1, m=0.1, \beta=0.1, x=0.8$. (k)- Variation of $u$ with y for different values of $m$ at $E_{1}=1, E_{2}=0.1, E_{3}=0.3, E_{4}=0.01, E_{5}=0.01, \mathrm{We}=0.03, \epsilon=0.2, t=0.02, M=1, k=1, \beta=$ $0.1, x=0.8$. (1)- Variation of $u$ with $\mathrm{y}$ for different values of $\beta$ at $E_{1}=1, E_{2}=0.1, E_{3}=0.3, E_{4}=$ $0.01, E_{5}=0.01$, We $=0.03, \epsilon=0.2, t=0.02, M=1, k=1, m=0.1, x=0.8$. (m)- Variation of $u$ with $\mathrm{y}$ for different values of $x$ at $E_{1}=1, E_{2}=0.1, E_{3}=0.3, E_{4}=0.01, E_{5}=0.01$, We $=0.03, \epsilon=$ $0.2, t=0.02, M=1, k=1, m=0.1, \beta=0.1$.
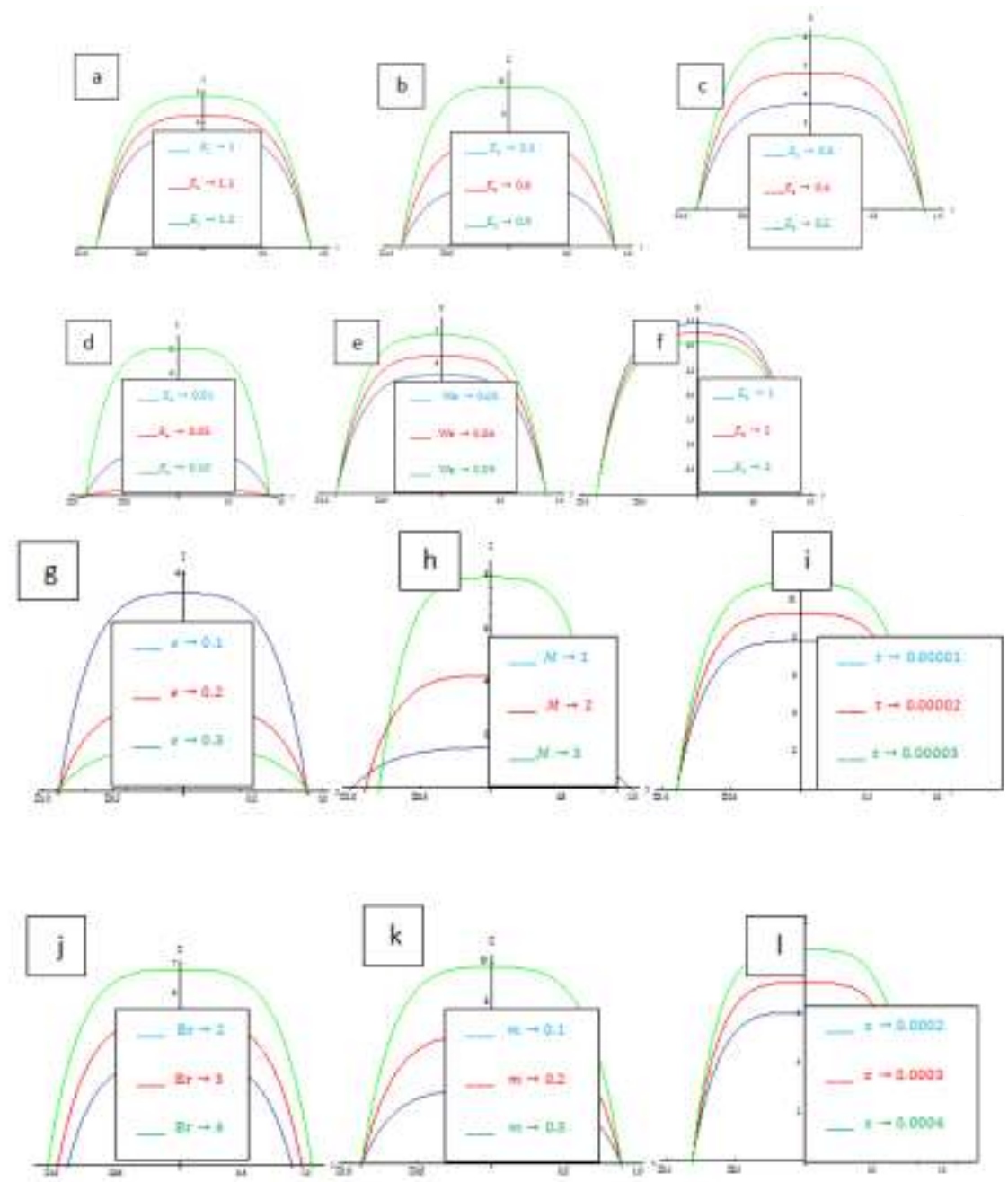

Figure 14.(a) Variation of $\theta$ with y for different values of $E_{1}$ at $E_{2}=0.3, E_{3}=0.3, E_{4}=0.01, E_{5}=0.01$, We $=0.03, \epsilon=0.2, t=0.02, M=1, m=0.1, x=0.8$. (b)- Variation of $\theta$ with y for different values of $E_{2}$ at $\quad E_{1}=1, E_{3}=0.3, E_{4}=0.01, E_{5}=0.01, \mathrm{We}=0.03, \epsilon=0.2, t=0.02, M=1, m=0.1, x=$ 0.8 . (c)- Variation of $\theta$ with y for different values of $E_{2}$ at $E_{1}=1, E_{3}=0.3, E_{4}=0.01, E_{5}=0.01, \mathrm{We}=$ $0.03, \epsilon=0.2, t=0.02, M=1, m=0.1, x=0.8$. (d)- Variation of $\theta$ with y for different values of $E_{4}$ at $E_{1}=1, E_{2}=0.1, E_{3}=0.3, E_{5}=0.01, \mathrm{We}=0.03, \epsilon=0.2, t=0.02, M=1, m=0.1, x=0.8$. (e)Variation of $\theta$ with y for different values of $W e$ at $E_{1}=1, E_{2}=0.1, E_{3}=0.3, E_{4}=0.01, E_{5}=0.01, \epsilon=$ $0.2, t=0.02, M=1, m=0.1, x=0.8$. (f)- Variation of $\theta$ with y for different values of $E_{5}$ at $E_{1}=1$, $E_{2}=0.1, E_{3}=0.3, E_{4}=0.01 w e=0.03, \epsilon=0.2, t=0.02, M=1, m=0.1, x=0.8$. (g)- Variation 
of $\theta$ with y for different values of $\epsilon$ at $E_{1}=1, E_{2}=0.1, E_{3}=0.3, E_{4}=0.01, E_{5}=0.01$, We $=0.03, t=$ $0.02, M=1, m=0.1, x=0.8$. (h)- Variation of $\theta$ with y for different values of $M$ at $E_{1}=1, E_{2}=$ $0.1, E_{3}=0.3, E_{4}=0.01, E_{5}=0.01, \mathrm{We}=0.03, \epsilon=0.2, t=0.02, m=0.1, x=0.8$. (i)- Variation of $\theta$ with $\mathrm{y}$ for different values of $t$ at $E_{1}=1, E_{2}=0.1, E_{3}=0.3, E_{4}=0.01, E_{5}=0.01, \mathrm{We}=0.03, \epsilon=$ $0.2, M=1, m=0.1, x=0.8$. (j)- Variation of $\theta$ with $y$ for different values of $\operatorname{Brat} E_{1}=1, E_{2}=$ $0.1, E_{3}=0.3, E_{4}=0.01, E_{5}=0.01, \mathrm{We}=0.03, \epsilon=0.2, t=0.02, M=1, m=0.1, x=0.8$. (k)Variation of $\theta$ with y for different values of $m$ at $E_{1}=1, E_{2}=0.1, E_{3}=0.3, E_{4}=0.01, E_{5}=0.01$, We = $0.03, \epsilon=0.2, t=0.02, M=1, B r=2, x=0.8$.(1)- Variation of $\theta$ with y for different values of $x$ at $E_{1}=1, E_{2}=0.1, E_{3}=0.3, E_{4}=0.01, E_{5}=0.01$, We $=0.03, \epsilon=0.2, t=0.02, M=1, m=0.1$, .

\section{Graphical Results And Discussion}

In this chapter, we have presented a set of figures to observe the behavior of sundry parameters involved in the expressions of longitudinal velocity $\left(u=\psi_{0 y}+W e \psi_{1 y}\right)$, temperature $\theta$ and stream function $\psi$. Figs 13.(a) to13.(m)display the effects of various physical parameters on the velocity profile $(y)$. Figs 13.(a) and 13.(b) depicts that the velocity increases when $E_{1}$ and $E_{2}$ enhanced .It due to the fact that less resistance is offered to the flow because of the wall elastance and thus velocity increases. However reverse effect is observed for $E_{3}, E_{4}$ and $E_{5}$. As $E_{3}$ represent the damping which is resistive force so velocity decreases when $E_{3}$ is increased. Similar behavior is observed for the velocity in case of rigidity and stiffness due to presence of damping force. Fig 13.(h)shows that the velocity decreases by increasing Hartman number . It is due to the fact that magnetic field applied in the trasverse direction shows damping effect on the flow. Fig 13.(f) illustrates that velocity profile decreases for $W e$ in the region $[-1,0]$ whereas it has opposite behavior in the region $[0,1]$. Figs 13.(j),13.(k) and 13.(l)depicts that the velocity increases when $m, k a n d \beta$ enhanced.Effects of pertinent parameters on temperature profile can be visualized throughFigs 14.(a) to14.(1). The variation of compliant wall parameters is studied in Figs13.(a) to 17.(e). As temperature is the average kinetic energy of the particles and kinetic energy depends upon the velocity. Therefore increase in velocity by $E_{1}$ and $E_{2}$ leads to temperature enhancement .Similarly decrease in velocity by $E_{3}, E_{4}$ and $E_{5}$ shows decay in temperature. Fig14.(h)reveals that the temperature profile $\theta$ decreases when Hartman number $M$ is increased .Effect of $B r$ on temperature can be observed through Fig14.(j). The Brinkman number $B r$ is the product of the Prandtl number $\operatorname{Pr}$ and the Eckert number Ec. Here Eckert number occurs due to the viscous dissipation effects and the temperatureenhances.Fig14.(k)depictthattheTemperatureincreaseswhenmenhanced.Thefunctionof an internally circulating bolus of fluid by closed stream lines is shown in Figs $\mathbf{1 2}$. Fig $\mathbf{1 0}$ depict that the size of trapped bolus increases when there is an increase in the Hartman number. We have analyzed from Figs $\mathbf{2}$ and $\mathbf{3}$ that the size of trapped bolus increases when $E_{1}$ and $E_{2}$ are enhanced. However it decreases when $E_{3}$ is increased. Also when we decrease the values of $E_{4} a n d E_{5}$ the trapped bolus size increases. Fig7 depict the stream lines pattern for different values of Weissenberg number.

\section{References}

[1]. K. Yazdanpanth-Ardakani and H. Niroomand-Oscii, New approach in modellingperistaltictransport of non-Newtonian fluid, J. Mech. Med. Biol., 13 (2013) 1350052.

[2]. A.M. Abd-Alla, S. M. Abo-Dahab and H.D.El-Shahrany, Effects of rotation and initialstress on peristaltic transport of fourth grade fluid with heat transfer and induced

[3]. magnetic field, J. Magn. Mater., 349 (2014) 268-280.

[4]. K. Nowar, Peristaltic flow of a nanofluid under the effect of Hall current and porousmedium, Math. Prob. Eng. (2014).

[5]. M. Kothandapani and J. Prakash, Effect of radiation and magnetic field on peristaltictransport of nanofluids through a porous space in a tapered asymmetric channel, J.Magn. Magn.Mater. 378 (2015) 152-163.29

[6]. G. Radhakrishnamacharya, Ch. Srinivasulu, Influence of wall properties on peristaltictransport with heat transfer, C. R. Mecanique 335 (2007) 369-373.

[7]. S. Srinivas, M. Kothandapani, The influence of heat and mass transfer on MHD peristalticflow through a porous space with compliant walls, Appl. Math. Comput. 213(2009) 197-208

[8]. H. Yasmin, T. Hayat, N. Alotaib and H. Gao, Convective heat and mass transfer analysison peristaltic flow of Williamson fluid

[9]. with Hall effects and Joule heating, Int. J.Biomath., DOI: 10.1142/S1793524514500582. 7 (2014).[8] T. Hayat, F. M. Abbasi, Maryem Al-Yami and ShathaMonaquel, Slip and Joule heatingeffects in mixed convection peristaltic transport of nanofluid with Soret and Dufoureffects, J. Mol. Liq., 194 (2014) 9399. [9] M. Mustafa, S. Abbasbandy, S. Hina and T. Hayat, Numerical investigation on mixedconvective peristaltic flow of fourth grade fluid with Dufour and Soret effect, J. Taiwan.Ins. Chem. Eng., 45 (2014) 308-316. 
[10]. M. Kothandapani and J. Prakash, Effects of thermal radiation parameter and magneticfield on the peristaltic motion of Williamson nanofluids in a tapered asymmetric channel,Int. J. Heat Mass Transfer, 81 (2015) 234-245.

[11]. Obaidullahmehmood, N. mustapha, S. Shafie and T. Hayat, Partial slip effect on heatand mass transfer of MHD peristaltic transport in a porous medium, Sains Malays. 43(2014) 1109-1118.

[12]. V. P. Rathod and L. Devindrappa, Peristaltic transport of a conducting fluid in an

[13]. asymmetric vertical channel with heat and mass transfer, J. Chem. Biol. Phys. Sci. 4 (2014) 1452-1470. 